\title{
Evaluating a person-centered chronic pain service using indices of reliable and clinically significant change
}

\author{
Katherine Macey BA (Hons) MSc DClinPsy ${ }^{a}$, Simon Clarke BSc PGCert DClinPsy ${ }^{b}$, Nima \\ Golijani-Moghaddam BSc (Hons) PhD DClinPsy ${ }^{c}$ and Roshan das Nair BA MSc MPhil PhD \\ CPsychol AFBPsS ${ }^{d}$
}

\begin{abstract}
a Clinical Psychologist, School of Medicine, University of Nottingham, Nottingham, UK
b Clinical Psychologist, Physical Health Clinical Psychology Services, Nottinghamshire Healthcare NHS Trust, Arthritis Research UK Pain Centre, University of Nottingham, Nottingham, UK

c Clinical Psychologist \& Research Tutor, School of Psychology, University of Lincoln, Brayford Pool, Lincoln, UK

d Research Tutor, School of Medicine, University of Nottingham \& Department of Clinical Psychology \& Neuropsychology, Nottingham University Hospitals NHS Trust, Nottingham, UK
\end{abstract}

\begin{abstract}
Background: Psychological interventions for chronic pain often require substantial individualisation, which can undermine links with the research evidence on which treatment is based. To ensure patients receive effective therapy, evaluation is needed. This paper demonstrates the use of individual level change indices, which may be more appropriate for assessing effectiveness of person-centred treatment than traditional group-level statistics.

Aims: To evaluate the efficacy of a psychological chronic pain service at individual level and to recommend improvements for future service evaluation.

Method: Indices of reliable and clinically significant change were used to assess outcomes on the HADS, CORE-10 and PSEQ.

Results: Fifty-six out of 83 patients had usable outcome data. On the most widely administered outcome measure, the CORE-10, $n=15$ met reliable change and $n=22$ met clinically significant change criteria. Eighteen showed no reliable change. Only one person deteriorated.

Conclusions: The person-centred treatment provided by this service was effective and achieved outcomes similar to published data from a structured group programme. However, missing data reduced the reliability of these conclusions and led to reduced usefulness of the evaluation for service planning. Recommendations for future evaluation are made.
\end{abstract}

\section{Keywords}

Audit, chronic pain, evidence-based practice, outcome measures, patient choice, patient experience, person-centered healthcare, practice-based evidence, psychology services, service evaluation, service effectiveness, shared decision-making

\section{Correspondence address}

Dr. Katherine Macey, School of Medicine, University of Nottingham, King’s Meadow Campus, Lenton Lane, Nottingham, NG7 2NR, UK. E-mail: kjm033@hotmail.co.uk

Accepted for publication: 21 March 2014

\section{Introduction}

This paper describes the process of evaluating a chronic pain psychology service using indices of reliable and clinically significant change to assess the efficacy of person-centred care provided to individual patients over a 12-month period. This means of evaluation overcomes some of the difficulties of using group-level statistics when evaluating individualised treatment and may be useful to other practitioners evaluating person-centred, individually tailored treatment packages. The primary aim of this study was to evaluate the effectiveness of the service using routinely collected outcome data. A secondary aim was to make recommendations for future service evaluation in order to make the process more useful for improving the service received by patients. This is part of a wider evaluation strategy involving formal and informal qualitative feedback to inform service development.

\section{Service Background}

The service provides individual and group psychological therapy to patients experiencing chronic pain, who have difficulties managing pain or the distress associated with their pain. Referral is mainly through hospital Consultants, but other professional groups (e.g., Pain Specialist Nurses) also refer. 
During the financial year in which this evaluation was conducted the service had undergone structural changes and a reduction in staff. They had also started to introduce different therapeutic modalities into their approaches, including so-called 'third-wave' cognitive approaches such as Acceptance Commitment Therapy (ACT), mindfulness and compassion-based approaches. The team were keen to maintain and improve quality and were considering changes to recording data and evaluation methods to facilitate this. The change to GP commissioning [1] means that demonstrating a commitment to evaluation and continuous improvement will be increasingly important. Demonstrable effectiveness and cost-effectiveness will be important for securing the tender for this service and tendering for other services in the future. It was hoped that evaluation would contribute to service planning and ultimately to better outcomes for the people using the service.

\section{Psychological treatment of pain}

Pain is a multi-factorial experience which can have a devastating impact on a person's life [2]. Pain and associated disability may cause or exacerbate anxiety and depression and anxiety and depression may in turn lead to increased experience of pain and further reductions in activity [3]. The impact of pain on a person's life is thought to be mediated by psychological factors such as catastrophising and mood, social factors such as partner support and behaviours such as coping strategies [4,5]. Psychologists in this service generally use cognitive behavioural (CBT) and, more recently, acceptance-based approaches to pain, which have been shown to be effective in meta-analyses of randomised controlled trials (RCTs) [6,7]. These approaches are combined with psychoeducation on chronic pain based on the outcomes of research on pain neurology [2,3]. However, chronic pain presentations can vary considerably and the evidence-base is not yet sufficiently developed to be able to specify which aspects of intervention are most appropriate for whom [8]. Therefore, the service maintains a flexible approach to treatment based on patient need.

As the service context is based within a general hospital location, the psychological service is often the patient's first ever contact with a mental health professional. Many patients find it hard to identify the psychological component to their pain and may struggle with stigma and perceptions of failure. Thus, the engagement of patients and the development of idiosyncratic, person-centred goals is prioritised over the rigid deployment of fixed protocols.

Individualised person-centred interventions are designed collaboratively with patients following assessment and formulation. CBT interventions include psycho-education, cognitive restructuring, goal-setting, activity scheduling, thought diaries and relaxation [9-11]. Acceptance-based approaches help individuals indentify and live by their values, employing techniques such as mindful-breathing. Learning to tolerate some level of pain, or at least accepting some of the limitations pain places on the individual, may allow patients to re-engage with meaningful and valued aspects of life that have become neglected in an unsuccessful attempt at pain relief or avoidance of the noxious experience of pain [7]. Patient choice is respected and patients can choose either individual or group treatment. Group sessions focus on topics such as psycho-education, values, goal-setting, activity-scheduling, mindful breathing and acceptance exercises within an acceptance-based CBT framework. The aim of treatment is to improve quality of life and reduce disability, even if the pain itself cannot be reduced.

\section{Evidence-based practice and Practice- based evidence}

Efforts to improve care and cost-efficiency have led to an increasing emphasis on evidence-based practice, that is, therapies supported by RCTs. However, while NICE guidelines summarise evidence for specific conditions, for example, low back pain [12], more research is needed into which interventions are most helpful to whom in the majority of pain conditions. While clinicians may have an interest in contributing to the evidence-base, the primary concern of most clinicians is to provide the best possible care they can to each individual patient.

There are several reasons why following standardised protocols may not lead to optimal treatment for individuals: RCTs can rely on highly selected samples, which may differ significantly from populations served by healthcare teams. Individual differences mean that even treatments that appear very successful when examined at the group level may not benefit all participants and some participants may even deteriorate [13]. Clinicians in this service, therefore, develop person-centred treatment plans within the context of an agreed and discussed formulation [14]. This is in line with available NICE guidance, which suggests adapting interventions for the individual where appropriate. This is particularly the case in complex longterm conditions such as pain.

Interventions adapted from the available evidence-base may differ importantly from the protocols they are derived from. A complimentary paradigm of practice-based evidence has therefore evolved to investigate the effectiveness of interventions as they are practiced [15]. Service evaluation is one form of practice-based evidence [16].

\section{Aims}

This service evaluation examined individual level outcomes of all patients discharged over a 12-month period. The main aim was to evaluate the effectiveness of the service each patient had received for the annual report. A secondary aim was to consider how future evaluation could be improved to make it more useful in terms of service planning. 


\section{Methods}

This service evaluation was approved under local governance procedures and informed by BPS ethical standards [17]. All measures were routinely collected by the service and no additional burden was placed on patients.

\section{Inclusion criteria}

Evaluation concerned performance over the financial year (FY) 2012-13. Clinical work runs continuously and patients referred towards the end of one FY or with complex difficulties may have therapy that spans more than one FY. In order to evaluate outcomes it was decided to identify patients discharged during FY 2012-13 irrespective of their referral date. The sample therefore contained patients referred prior to 2012-13 and excluded patients referred during the FY who had not yet completed therapy. Eighty-three patients met this criterion.

\section{Audit Sheets}

Clinicians completed 'Audit Sheets' designed by the service for each of their discharged patients. The Audit Sheets requested information regarding gender, referrer, presenting problem, presence of anxiety and depression, number of sessions, psychometric outcome data and functional outcome (e.g., back in work, education), reasons for missing data and also had room for comments.

\section{Outcome measures}

\section{The CORE-10}

The CORE-10 is an abbreviated version of the Clinical Outcomes in Routine Evaluation Outcome Measure (CORE-OM [18]). The CORE-OM was developed through consultation with professionals working from a range of models [19]. The CORE-10 comprises 10 items assessing anxiety, depression, trauma, physical problems, functioning and risk. It can be used as a session-by-session rating scale or as a quick outcome measure. It has been shown to be sensitive to change and to correlate highly with the 28-item version of the scale $(r=0.94$ in a clinical sample) and has good internal consistency ( $\alpha=0.82)$ [20].

\section{HADS}

The Hospital Anxiety and Depression Scale (HADS) [21] comprises two 7-item scales (anxiety and depression) with items rated on a 4-point scale. Developed as a screening tool for depression and anxiety in hospital out-patients, it has since been used extensively in primary care and mental health settings [22] and in research [22,23]. The HADS has been widely translated and used across cultures, has been found to correlate with other measures of anxiety and depression, is sensitive to change and has good internal reliability ( $\alpha=0.80$ to 0.93 for the anxiety and 0.81 to 0.90 for the depression subscales) [24].

\section{PSEQ}

The Pain Self-efficacy Questionnaire (PSEQ [25]) contains 10 items. Respondents rate their belief in their ability to complete different activities despite their pain on a 7-point scale ranging from $0=$ 'not at all confident' to 6='completely confident'. Pain self-efficacy has been shown to correlate with lower levels of pain and distress and with a greater ability to tolerate painful stimuli. Increases in self-efficacy have been associated with positive outcomes in pain-coping interventions [4]. The PSEQ has high internal consistency $(\alpha=0.92)$ and has demonstrated sensitivity to treatment effects across numerous studies.

\section{Analyses}

A trainee clinical psychologist entered the data from Audit Sheets into an anonymised Excel spreadsheet and used IBM SPSS Version 20 for analysis. Outcomes were assessed using single-case statistical techniques: reliable change index, clinically significant change cut-offs and where possible benchmarking against published outcomes.

\section{Reliable and clinically signific ant change criteria}

The Reliable Change Index (RCI) was used to, 'determine whether the magnitude of change for a given patient [was] statistically reliable' [26]. That is, to ascertain whether the change was greater than could be expected from measurement error and artefacts of repeated measurement. Established criteria were used: for the HADS and the PSEQ the RCI calculated by Morley, Williams, \& Hussain [27] was used; for the CORE-10 the RCI derived by the scale's authors was used [20].

Clinically Significant Change (CSC) cut-off criteria were used to determine whether individual patients who showed reliable change could be said to have 'recovered' following therapy. As with the RCI, CSC criteria established by $[20,27]$ were adopted. See Table 1 .

Table 1 Table depicting RCI and CSC Criteria

\begin{tabular}{lcc}
\hline Measure & $\begin{array}{c}\text { Reliable Change } \\
\text { Index }(\mathrm{RCI}) \text { value }\end{array}$ & $\begin{array}{c}\text { Clinically Significant } \\
\text { Change cut-off (CSC) }\end{array}$ \\
\hline CORE-10 & 6 & $<11$ \\
HADS-Anxiety & 4.77 & $<8$ \\
HADS-Depression & 4.58 & $<8$ \\
PSEQ & 8.23 & 43.8 and above \\
\hline
\end{tabular}




\section{Results}

\section{Available data}

Eighty-three patients were discharged during the financial year 2012-13; 13.49 (59\%) were female, 30 (36\%) were male. For 4 patients gender was not recorded. No other demographic variables were recorded. Information regarding the number of sessions attended was available for 66 patients; the mean number of sessions was 9.89 ranging from 2-60 sessions. Mean scores on pre-treatment measures indicated moderately high levels of anxiety, depression and general distress. See Table 2.

Table 2 Table showing descriptive statistics for pre-treatment measures and number of sessions

\begin{tabular}{lccccc}
\hline & $\mathrm{n}$ & Minimum & Maximum & Mean & $\begin{array}{c}\text { Standard } \\
\text { Deviation }\end{array}$ \\
\hline HADS Anxiety & 38 & 4 & 21 & 14.5 & 4.1 \\
HADS Depression & 38 & 1 & 21 & 13.6 & 4.6 \\
CORE-10 & 76 & 0 & 38 & 23.6 & 7.6 \\
PSEQ & 15 & 0 & 41 & 13.5 & 10.9 \\
Total Sessions & 66 & 0 & 50 & 9.9 & 8.5 \\
\hline
\end{tabular}

\section{Outcome data}

Fifty-six patients had pre- and post-therapy scores for the CORE-10; 11 patients had pre- and post-therapy scores for the HADS and 7 patients had pre- and post-scores for the PSEQ. For 27 patients (32.5\%) there were no available outcome data. For 56 patients (67.5\%), there was at least one measure that could be used to evaluate therapeutic progress.

\section{Reasons for missing data}

Reasons for missing data were given for 11 patients (41\% of those with missing data; $13 \%$ of total sample). Reasons included: unplanned endings such as loss of contact or missed final appointment $(\mathrm{n}=8)$, indirect working / no direct contact $(n=1)$, or the patient did not speak English $(n=2)$. However, for 16 patients (59\% of those with missing data; $19 \%$ of the sample) no reason was recorded. Between samples t-tests revealed no significant differences between the pre-therapy scores of those patients who did and did not have outcome (post-therapy) data. Patients were categorised according to outcome in the following categories shown in Box 1.

\section{Box 1 Criteria employed to categorise patients according to outcome}

Clinically significant improvement - improvement that met both RCI and CSC criteria

Reliable improvement - improvement that met $\mathrm{RCI}$ but not CSC criteria

No change - magnitude of any change following treatment was within range expected due to measurement error

Reliable deterioration - deterioration that met $\mathrm{RCl}$ criterion but not criterion for CSC

Clinically significant deterioration - deterioration that met both $\mathrm{RCI}$ and CSC criteria

The number and proportion of patients within each category for each outcome measure is shown in Table 3. The majority of participants met RCI or CSC criteria. However, for some participants changes could not be distinguished from measurement error. One patient showed reliable deterioration on the CORE-10, however, no patients showed clinically significant deterioration.

Table 3 Table showing number of patients meeting different outcome criteria for each measure

\begin{tabular}{l|ccccc}
\hline N & RCI & CSC & $\begin{array}{c}\text { No } \\
\text { change }\end{array}$ & $\begin{array}{c}\text { Reliable } \\
\text { Deterioration }\end{array}$ & $\begin{array}{c}\text { No } \\
\text { data }\end{array}$ \\
\hline HADS A & 2 & 3 & 6 & 0 & 72 \\
HADS D & 3 & 3 & 5 & 0 & 72 \\
PSEQ & 4 & 0 & 3 & 0 & 76 \\
CORE-10 & 15 & 22 & 18 & 1 & 27 \\
\hline
\end{tabular}

The following graph (Figure 1.) illustrates the proportion of patients meeting each outcome criterion.

Figure 1 Bar graph showing proportion (\%) of patients meeting criteria for different categories at discharge

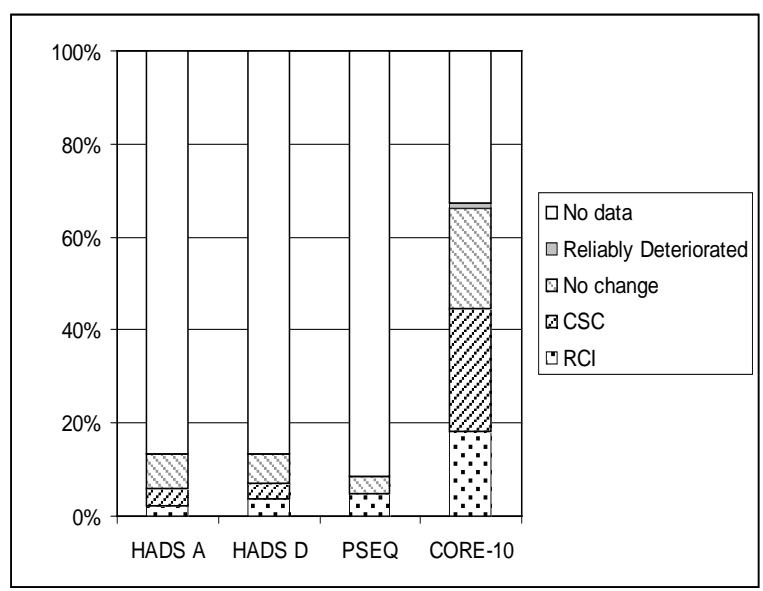




\section{Benchmarking}

These figures were compared to the HADS and PSEQ results achieved by Morley et al. [27] and, in line with this paper, only those patients for whom outcome data were available were included in the analyses. Benchmark figures for the CORE-10 in chronic pain interventions could not be located. (See Table 4).

Table 4 Table showing benchmark comparisons

\begin{tabular}{|c|c|c|c|}
\hline & & Proportion & patients (\%) \\
\hline Measure & Outcome & $\begin{array}{c}\text { Current } \\
\text { evaluation }\end{array}$ & $\begin{array}{c}\text { Benchmark } \\
\text { evaluation } \\
\text { (Morley et } \\
\text { al. [27]) }\end{array}$ \\
\hline \multirow[t]{4}{*}{ HADS A } & $\begin{array}{l}\text { Clinically significant } \\
\text { improvement }\end{array}$ & 27 & 14 \\
\hline & $\begin{array}{l}\text { Reliable } \\
\text { Improvement }\end{array}$ & 18 & 10 \\
\hline & No change & 55 & 71 \\
\hline & Reliable Deterioration & 0 & 4 \\
\hline \multirow[t]{4}{*}{ HADS D } & $\begin{array}{l}\text { Clinically significant } \\
\text { improvement }\end{array}$ & 27 & 18 \\
\hline & $\begin{array}{l}\text { Reliable } \\
\text { Improvement }\end{array}$ & 27 & 8 \\
\hline & No change & 45 & 68 \\
\hline & Reliable Deterioration & 0 & 6 \\
\hline \multirow[t]{4}{*}{ PSEQ } & $\begin{array}{l}\text { Clinically significant } \\
\text { improvement }\end{array}$ & 0 & 17 \\
\hline & $\begin{array}{l}\text { Reliable } \\
\text { Improvement }\end{array}$ & 57 & 33 \\
\hline & No change & 43 & 44 \\
\hline & Reliable Deterioration & 0 & 6 \\
\hline
\end{tabular}

\section{Disc ussion}

Outcome data indicate that the majority of patients benefitted from treatment. However some were still symptomatic at discharge and one patient showed deterioration. It is not possible to assess the impact of extra-therapeutic factors, such as life circumstances, on these results. Despite this, the results appear encouraging and compare favourably to available benchmarks.

There are several limitations to our study, including missing data and the measures used that mean results should be interpreted with caution. Patients who drop out of therapy, or who have unplanned endings, may have poorer outcomes [28] and nearly a fifth of the sample had no explanation for missing outcome data. These factors may have contributed to an inflated picture of service effectiveness.

Completion rates for outcome measures vary across services. In this sample, $67.5 \%$ of patients completed at least one post-therapy measure. This compares favourably with a mean of $39 \%$ in a recent investigation into completion rates [28]. Use of routine measures may be inappropriate for some patients, for example, due to language barriers, lack of appropriate norms, or patients appearing distressed or fatigued by them. Some patients, particularly in a physical healthcare environment, may not be familiar with completing measures about their mood and some may refuse to complete them for reasons of feeling stigmatised. However, measures can also elicit useful information and enhance rapport [29].

Clinicians need flexibility to depart from standardised measures where these would jeopardise engagement, or lack validity. However, some form of evaluation is necessary to ensure that patients receive effective therapy. Consistent recording of reasons for not using measures may lead to insights into how person-centred evaluation can be developed in the service, for example, use of personal Goal Attainment Scaling [30].

Partially completed Audit Sheets resulted in information from patient files not being communicated to the researchers and this impacted on the usefulness of the evaluation for service improvement. For example, for the majority of patients, it was not clear who had received group therapy, individual therapy, or both. Therefore, the relative effectiveness of different formats (which could have implications for service planning) could not be evaluated. Similarly, functional outcome was rarely recorded. Reading files is regarded as 'processing' under the Data Protection Act (2003) of the UK, so allowing third party access to patient files in future would require careful ethical consideration and potentially more complex and time-consuming ethical processes [17].

The ease of administration of brief measures such as the CORE-10 and HADS comes at the cost of reduced diagnostic specificity and sensitivity [29,31,32]. In light of the British Psychological Society Division of Clinical Psychology's position that individual and contextualised accounts of distress are more useful than psychiatric diagnoses [33] this may seem unproblematic and, despite their limitations, the HADS and CORE-10 may provide useful indices of general distress [34]. However, it has been suggested that the HADS item "I feel as if I am slowed down" should be removed in samples where it may be confounded by symptoms of physical illness [35]. There may also be significant overlap between the HADS and the CORE-10, which both measure generalised psychological distress.

Use of session-by-session measures such as the Outcome Rating Scale (ORS) and the Session Rating Scales (SRS) [36,37] may have the advantage of allowing assessment of change when therapy ends prematurely. They may even indicate risk of early drop-out in time to prevent this. 


\section{Conclusions and recommendations}

Conclusions of future evaluations will be more robust if the proportion of patients with both pre- and post-therapy data could be increased. Decisions not to use measures should be recorded clearly and alternative nonstandardised measures should be considered. Recording numbers of sessions missed and attended would allow for assessment of engagement and for a distinction to be made between the service offered and service utilisation itself. Recording group attendance separately from individual sessions would also make evaluation more useful in practical terms. Recording demographic factors such as age may lead to identification of groups experiencing less benefit and where there is a need for service adaptation. Guidelines concerning clinical trials suggest measures of subjective pain intensity and behavioural outcomes should also be considered to clarify how treatment affects pain and behaviour [38]. It is hoped that making these improvements in evaluation will allow the service to identify relative strengths and weaknesses in order to improve overall patient experience and contribute to the development of more effective psychological treatments for chronic pain.

\section{Conflicts of Interest}

The authors declare no conflicts of interest.

\section{References}

[1] Department of Health (DOH). (2010). Equity and excellence: Liberating the NHS. London: Department of Health.

[2] Melzack, R. (2001). Pain and the neuromatrix in the brain. Journal of Dental Education 65 (12) 1378-1382.

[3] Wiech, K. \& Tracey, I. (2009). The influence of negative emotions on pain: behavioral effects and neural mechanisms. NeuroImage 47 (3) 987-994.

[4] Keefe, F.J., Rumble, M.E., Scipio, C.D., Giordano, L.A. \& Perri, L.M. (2004). Psychological aspects of persistent pain: current state of the science. Journal of Pain 5 (4) $195-211$.

[5] Turk, D.C. \& Okifuji, A. (2002). Psychological Factors in Chronic Pain: Evolution and Revolution. Journal of Consulting and Clinical Psychology 70 (3) 678-690.

[6] Morley, S., Eccleston, C. \& Williams, A. (1999). Systematic review and meta-analysis of randomized controlled trials of cognitive behaviour therapy and behaviour therapy for chronic pain in adults, excluding headache. Pain 80, 1-13.

[7] Veehof, M.M., Oskam, M.-J., Schreurs, K.M.G. \& Bohlmeijer, E.T. (2011). Acceptance-based interventions for the treatment of chronic pain: a systematic review and meta-analysis. Pain 152 (3) 533-542.

[8] Jensen, M.P. \& Turk, D.C. (2014). Contributions of psychology to the understanding and treatment of people with chronic pain: Why it matters to ALL psychologists. American Psychologist 69 (2) 105-118.

[9] Lohnberg, J.A. (2007). A Review of Outcome Studies on Cognitive-Behavioral Therapy for Reducing FearAvoidance Beliefs Among Individuals With Chronic Pain. Journal of Clinical Psychology in Medical Settings 14 (2) 113-122.

[10] Turner, J.A, Holtzman, S. \& Mancl, L. (2007). Mediators, moderators, and predictors of therapeutic change in cognitive-behavioral therapy for chronic pain. Pain 127 (3) 276-286.

[11] Turner, J.A, Mancl, L. \& Aaron, L.A. (2006). Shortand long-term efficacy of brief cognitive-behavioral therapy for patients with chronic temporomandibular disorder pain: a randomized, controlled trial. Pain 121 (3) 181-194.

[12] NICE. (2009). Early management of persistent nonspecific low back pain. London: NICE.

[13] Schulte, D. \& Bochum, R. (2002). What to Do When Manuals Fail ? The Dual Model of Psychotherapy. Clinical Psychology: Science and Practice 9 (3) 312-328.

[14] British Psychological Society (BPS). (2011). Good Practice Guidelines on the use of psychological formulation. Leicester: British Psychological Society.

[15] Barkham, M. \& Mellor-Clark, J. (2003). Bridging evidence-based practice and practice-based evidence: developing a rigorous and relevant knowledge for the psychological therapies. Clinical Psychology \& Psychotherapy 10 (6) 319-327.

[16] Andrews, W., Twigg, E., Minami, T. \& Johnson, G. (2011). Piloting a practice research network: a 12-month evaluation of the Human Givens approach in primary care at a general medical practice. Psychology and Psychotherapy 84 (4) 389-405.

[17] Cooper, M., Turpin, G., Bucks, R. \& Kent, G. (2005). Good Practice Guidelines for the Conduct of Psychological Research within the NHS. Leicester: British Psychological Society.

[18] Evans, C., Mellor-Clark, J., Margison, F., Barkham, M., Audin, K., Connell, J. \& McGrath, G. (2000). CORE: Clinical Outcomes in Routine Evaluation. Journal of Mental Health 9 (3) 247-255.

[19] Mellor-Clark, J. (2007). CORE: A Decade of Development. Rugby: CORE IMS.

[20] Connell, J. \& Barkham, M. (2007). CORE-10 User Manual, Version 1.1. Rugby, UK: CORE Systems Trust \& CORE Information Management Systems Ltd.

[21] Zigmond, A.S. \& Snaith, R.P. (1983). The hospital anxiety and depression scale. Acta Psychiatrica Scandinavica 67 (6) 361-370.

[22] Bjelland, I., Dahl, A.A, Haug, T.T. \& Neckelmann, D. (2002). The validity of the Hospital Anxiety and Depression Scale. An updated literature review. Journal of Psychosomatic Research 52 (2) 69-77.

[23] Snaith, R.P. (2003). The Hospital Anxiety and Depression Scale. Health and Quality of Life Outcomes 4, 6-9.

[24] Herrmann, C. (1997). International experiences with the Hospital Anxiety and Depression Scale - a review of validation data and clinical results. Journal of Psychosomatic Research 42 (1) 17-41. 
[25] Nicholas, M.K. (2007). The pain self-efficacy questionnaire: Taking pain into account. European Journal of Pain 11 (2) 153-163.

[26] Jacobson, N.S. \& Truax, P. (1991). Clinical Significance: A Statistical Approach to Defining Meaningful Change in Psychotherapy Research. Journal of Consulting and Clinical Psychology 59 (1) 12-19.

[27] Morley, S., Williams, A. \& Hussain, S. (2008). Estimating the clinical effectiveness of cognitive behavioural therapy in the clinic: Evaluation of a CBT informed pain-management programme. Pain 137, 670680.

[28] Bewick, B., Trusler, K., Mullin, T., Grant, S. \& Mothersole, G. (2006). Routine outcome measurement completion rates of the CORE-OM in primary care psychological therapies and counselling. Counselling and Psychotherapy Research 6 (1) 33-40.

[29] Halstead, J., Youn, S.J. \& Armijo, I. (2013). Scientific and clinical considerations in progress monitoring: When is a brief measure too brief? Canadian Psychology / Psychologie Canadienne 54 (1) 83-85.

[30] Ottenbacher, K.J. \& Cusick, A. (1990). Goal Attainment Scaling as a method of Clinical Service Evaluation. American Journal of Occupational Therapy 44 (6) $519-525$.

[31] Cosco, T.D., Doyle, F., Ward, M. \& McGee, H. (2012). Latent structure of the Hospital Anxiety And Depression Scale: a 10-year systematic review. Journal of Psychosomatic Research 72 (3) 180-184.

[32] Coyne, J.C. \& van Sonderen, E. (2012). The Hospital Anxiety and Depression Scale (HADS) is dead, but like Elvis, there will still be citings. Journal of Psychosomatic Research 73 (1) 77-78.

[33] DCP. (2013). Classification of behaviour and experience in relation to functional psychiatric diagnoses: Time for a paradigm shift - Position Statement. Leicester: British Psychological Society.

[34] Norton, S., Cosco, T., Doyle, F., Done, J. \& Sacker, A. (2013). The Hospital Anxiety and Depression Scale: a meta confirmatory factor analysis. Journal of Psychosomatic Research 74 (1) 74-81.

[35] Gibbons, C.J., Mills, R.J., Thornton, E.W., Ealing, J., Mitchell, J.D., Shaw, P.J., Talbot, K., Tennant, A. \& Young, C.A. (2011). Rasch analysis of the hospital anxiety and depression scale (HADS) for use in motor neurone disease. Health and Quality of Life Outcomes 9 (1) 82.

[36] Duncan, B.L., Miller, S.D., Sparks, J.A., Claud, D.A., Beach, P., Reynolds, L.R. \& Johnson, L.D. (2003). The Session Rating Scale: Preliminary Psychometric Properties of a “ Working” Alliance Measure. Journal of Brief Therapy 3 (1) 3-12.

[37] Miller, S.D., Duncan, B.L., Sparks, J.A. \& Claud, D.A. (2003). The Outcome Rating Scale: A Preliminary Study of the Reliability, Validity and Feasibility of a Brief Visual Analog Measure. Journal of Brief Therapy 2 (2) 91100.

[38] Dworkin, R.H., Turk, D.C., Farrar, J.T., Haythornthwaite, J.A., Jensen, M.P., Katz, N.P. et al. (2005). Core outcome measures for chronic pain clinical trials: IMMPACT recommendations. Pain 113 (1-2) 9-19. 